\title{
Appropriate Similarity Measures for Author Cocitation Analysis
}

\author{
Nees Jan van Eck and Ludo Waltman
}

\begin{tabular}{|l|l|}
\hline \multicolumn{2}{|l|}{ ERIM REPORT SERIES RESEARCH IN MANAGEMENT } \\
\hline ERIM Report Series reference number & ERS-2007-091-LIS \\
\hline Publication & December 2007 \\
\hline Number of pages & 12 \\
\hline Persistent paper URL & http://hdl.handle.net/1765/10889 \\
\hline Email address corresponding author & Iwaltman@few.eur.nl \\
\hline Address & Erasmus Research Institute of Management (ERIM) \\
& RSM Erasmus University / Erasmus School of Economics \\
& Erasmus Universiteit Rotterdam \\
& P.O.Box 1738 \\
& 3000 DR Rotterdam, The Netherlands \\
& Phone: $\quad+31104081182$ \\
& Fax: $\quad+31104089640$ \\
& Email: info@erim.eur.nl \\
& Internet: $\quad$ www.erim.eur.nl \\
\hline
\end{tabular}

Bibliographic data and classifications of all the ERIM reports are also available on the ERIM website: www.erim.eur.nl 


\section{ERASMUS RESEARCH INSTITUTE OF MANAGEMENT}

\section{REPORT SERIES}

\section{RESEARCH IN MANAGEMENT}

\begin{tabular}{|l|l|}
\hline \multicolumn{2}{|l|}{ ABSTRACT AND KEYWORDS } \\
\hline Abstract & $\begin{array}{l}\text { We provide a number of new insights into the methodological discussion about author cocitation } \\
\text { analysis. We first argue that the use of the Pearson correlation for measuring the similarity } \\
\text { between authors' cocitation profiles is not very satisfactory. We then discuss what kind of } \\
\text { similarity measures may be used as an alternative to the Pearson correlation. We consider three } \\
\text { similarity measures in particular. One is the well-known cosine. The other two similarity } \\
\text { measures have not been used before in the bibliometric literature. Finally, we show by means of } \\
\text { an example that our findings have a high practical relevance. }\end{array}$ \\
\hline Free Keywords & \begin{tabular}{l} 
author cocitation analysis, similarity measure, Pearson correlation, cosine, information science \\
\hline Availability
\end{tabular} \\
$\begin{array}{l}\text { The ERIM Report Series is distributed through the following platforms: } \\
\text { Academic Repository at Erasmus University (DEAR), DEAR ERIM Series Portal } \\
\text { Social Science Research Network (SSRN), SSRN ERIM Series Webpage } \\
\text { Research Papers in Economics (REPEC), REPEC ERIM Series Webpage }\end{array}$ \\
\hline Classifications & $\begin{array}{l}\text { The electronic versions of the papers in the ERIM report Series contain bibliographic metadata } \\
\text { by the following classification systems: } \\
\text { Library of Congress Classification, (LCC) LCC Webpage } \\
\text { Journal of Economic Literature, (JEL), JEL Webpage } \\
\text { ACM Computing Classification System CCS Webpage } \\
\text { Inspec Classification scheme (ICS), ICS Webpage }\end{array}$ \\
\hline
\end{tabular}




\title{
Appropriate Similarity Measures for Author Cocitation Analysis
}

\author{
Nees Jan van Eck \\ Ludo Waltman \\ Econometric Institute, Erasmus School of Economics \\ Erasmus University Rotterdam \\ P.O. Box 1738, 3000 DR Rotterdam, The Netherlands \\ E-mail: \{nvaneck,lwaltman\}@few.eur.nl
}

\begin{abstract}
We provide a number of new insights into the methodological discussion about author cocitation analysis. We first argue that the use of the Pearson correlation for measuring the similarity between authors' cocitation profiles is not very satisfactory. We then discuss what kind of similarity measures may be used as an alternative to the Pearson correlation. We consider three similarity measures in particular. One is the well-known cosine. The other two similarity measures have not been used before in the bibliometric literature. Finally, we show by means of an example that our findings have a high practical relevance.
\end{abstract}

\section{Keywords}

Author cocitation analysis, similarity measure, Pearson correlation, cosine, information science.

\section{Introduction}

In the past few years, there has been a lot of discussion about the way in which author cocitation studies should be performed. Ahlgren, Jarneving, and Rousseau [1] questioned the appropriateness of the Pearson correlation for measuring the similarity between authors' cocitation profiles. Their paper caused quite some debate. In particular, White [8] argued that the objections 
of Ahlgren et al. against the Pearson correlation are mainly of theoretical interest and have little practical relevance. Leydesdorff and Vaughan [3], however, went even further than Ahlgren et al. and asserted that cocitation data should be analyzed directly, without first calculating a similarity measure. This is a point of view with which we do not agree [7]. Schneider and Borlund [6] pointed out that from a statistical perspective the common practice of calculating similarity measures based on cocitation profiles is rather unorthodox. In addition, they also mentioned some drawbacks of the use of the Pearson correlation as a similarity measure.

In this report, our aim is to provide a number of new insights into the above discussion. First of all, we agree with Schneider and Borlund [6] that from a statistical perspective calculating similarity measures based on cocitation profiles is a somewhat unconventional procedure. Although the procedure is unconventional, we do not believe that it has any fundamental statistical problems. Following Ahlgren et al. [1], we do believe that the use of the Pearson correlation to measure the similarity between cocitation profiles is problematic. Below, we will discuss some shortcomings of the Pearson correlation, most of which have not been mentioned before in the bibliometric literature. Because of these shortcomings, the Pearson correlation is, in our opinion, not a very satisfactory similarity measure for cocitation profiles. We will also discuss what kind of similarity measures may be used as an alternative to the Pearson correlation. Using a well-known author cocitation study by White and McCain [10] as an example, we will show that our findings are not merely of theoretical interest but also have a high practical relevance.

\section{Shortcomings of the Pearson correlation}

Suppose that we have a bibliographic data set and that we are interested in analyzing the cocitations of a set of $n$ authors in this data set. Typically, the analysis is performed as follows (for more details, see [5]). First, for each pair of two authors $i$ and $j$, the number of cocitations in the data set, denoted by $c_{i j}$, is counted. Next, the cocitation counts are used to calculate similarities between the authors. In most cases, this is done using the Pearson correlation as similarity measure for cocitation profiles. The similarity between authors $i$ and $j$ then has a value between -1 and 1 and is calculated as

$$
r(i, j)=\frac{\sum_{k \neq i, j}\left(c_{i k}-\bar{c}_{i}\right)\left(c_{j k}-\bar{c}_{j}\right)}{\sqrt{\sum_{k \neq i, j}\left(c_{i k}-\bar{c}_{i}\right)^{2} \sum_{k \neq i, j}\left(c_{j k}-\bar{c}_{j}\right)^{2}}},
$$


where $\bar{c}_{i}$ and $\bar{c}_{j}$ denote the averages of, respectively, the cocitation counts $c_{i k}$ and the cocitation counts $c_{j k}$ (for $k \neq i, j$ ). As a final step, the similarities between the authors are visualized using multidimensional scaling. ${ }^{1}$

We will now discuss some shortcomings of the Pearson correlation as a similarity measure for cocitation profiles. In the examples that we give, there are $n=6$ authors. Hence, when comparing two authors, each author's cocitation profile consists of four cocitation counts. Consider first the comparison between two authors, author 1 and author 2, with cocitation profiles

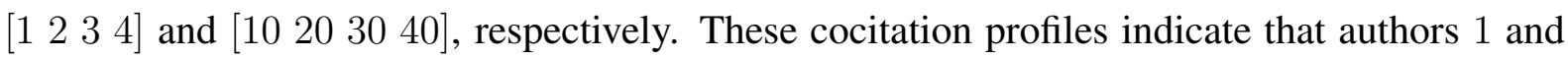
2 have, respectively, 1 and 10 cocitations with author 3,2 and 20 cocitations with author 4 , and so on. Although author 2 has ten times as many cocitations as author 1 , the relative frequencies with which authors 1 and 2 are cocited with each of the four other authors are exactly equal. That is, authors 1 and 2 both have $10 \%$ of their cocitations with author 3, $20 \%$ of their cocitations with author 4 , and so on. Since the similarity between two authors should not be influenced by each author's total number of cocitations, authors 1 and 2 should be regarded as perfectly similar. The Pearson correlation does indeed indicate a perfect similarity between the authors, as it has a value of 1 for the above two cocitation profiles. Now consider what happens when author 2's cocitation profile is changed into [11 1213 14]. The Pearson correlation still has a value of 1 , which again indicates a perfect similarity between the authors. However, whereas author 1 still has $10 \%$ of his cocitations with author $3,20 \%$ of his cocitations with author 4 , and so on, author 2 now has his cocitations more or less equally distributed. The cocitation profiles of authors 1 and 2 are therefore quite different, and the Pearson correlation incorrectly indicates a perfect similarity between the authors.

Another interesting example is obtained when authors 1 and 2 have cocitation profiles $\left[\begin{array}{llll}1 & 1 & 1 & 0\end{array}\right]$ and $\left[\begin{array}{llll}0 & 0 & 1 & 10\end{array}\right]$, respectively. In this example, there is no author with whom authors 1 and 2 have both been cocited. We would therefore expect the similarity between authors 1 and 2 to be as low as possible. However, the Pearson correlation has a value of -0.43 , which indicates a low similarity between the authors but not the lowest possible similarity. Now consider the following example. Authors 1 and 2 have cocitation profiles [ [ $\left.\begin{array}{llll}1 & 2 & 3 & 4\end{array}\right]$ and [ [ $\left.\begin{array}{llll}4 & 3 & 2 & 1\end{array}\right]$, respectively. In this example, the Pearson correlation has a value of -1 , and hence the similarity between

\footnotetext{
${ }^{1}$ We have not defined $c_{i j}$ for $i=j$. In the above equation, the Pearson correlation is therefore applied to cocitation profiles of length $n-2$ rather than length $n$. The diagonal elements of the cocitation matrix can also be handled in other ways, but this is not important for the present discussion.
} 
the authors is as low as possible. Comparing the last two examples, we believe that the Pearson correlation gives unsatisfactory results. In the first example, authors 1 and 2 do not have the lowest possible similarity, even though there is no author with whom they have both been cocited. In the second example, on the other hand, authors 1 and 2 do have the lowest possible similarity, even though they have both been cocited with all four other authors. In other words, in the second example authors 1 and 2 are regarded as less similar than in the first example, even though they have more similar distributions of their cocitations.

Based on the above examples, we believe that an appropriate similarity measure for cocitation profiles should at least satisfy the following two conditions:

1. The similarity between two authors is maximal if and only if the authors' cocitation profiles differ by at most a multiplicative constant.

2. The similarity between two authors is minimal if and only if there is no author with whom the two authors have both been cocited.

The above examples have shown that the Pearson correlation satisfies neither of these conditions. In our opinion, the Pearson correlation is therefore not a very satisfactory similarity measure for cocitation profiles.

\section{Alternatives to the Pearson correlation}

In addition to the Pearson correlation, the cosine is a relatively popular similarity measure for cocitation profiles. Using the cosine, the similarity between authors $i$ and $j$ has a value between 0 and 1 and is calculated as

$$
\cos (i, j)=\frac{\sum_{k \neq i, j} c_{i k} c_{j k}}{\sqrt{\sum_{k \neq i, j} c_{i k}^{2} \sum_{k \neq i, j} c_{j k}^{2}}} .
$$

Unlike the Pearson correlation, the cosine satisfies the two conditions introduced at the end of the previous section (for a discussion of the relationship between the Pearson correlation and the cosine, see [6]). Both the Pearson correlation and the cosine have the property that multiplying an author's cocitation profile by an arbitrary constant has no effect on the author's similarity with other authors. This is called the property of coordinate-wise scale invariance by Ahlgren et al. [1]. It is an indispensable property for any similarity measure for cocitation profiles, since 
it guarantees that the similarity between two authors is not influenced by each author's total number of cocitations. In other words, it guarantees that the similarity between two authors depends only on the relative frequencies with which the authors are cocited with other authors.

Because of the property of coordinate-wise scale invariance, the similarity between two authors calculated using a measure such as the Pearson correlation or the cosine does not change when the authors' cocitation profiles are normalized to sum to one. That is, the values of the Pearson correlation and the cosine do not change when the $c_{i k} \mathrm{~s}$ and $c_{j k} \mathrm{~s}$ in the equations provided above are replaced by $p_{i k} \mathrm{~s}$ and $p_{j k} \mathrm{~s}$ that are given by

$$
p_{i k}=\frac{c_{i k}}{\sum_{k^{\prime} \neq i, j} c_{i k^{\prime}}} \quad \text { and } \quad p_{j k}=\frac{c_{j k}}{\sum_{k^{\prime} \neq i, j} c_{j k^{\prime}}} .
$$

Interestingly, these $p_{i k} \mathrm{~s}$ and $p_{j k} \mathrm{~s}$ have a natural interpretation in probabilistic terms. $p_{i k}\left(p_{j k}\right)$ can be interpreted as the probability that a randomly drawn cocitation of author $i(j)$ is a cocitation with author $k$. Under this interpretation, the normalized cocitation profile of author $i(j)$ is a probability distribution that indicates the probability of author $i(j)$ being cocited with each of the other authors. Hence, when we are comparing the cocitation profiles of two authors, what we are in fact doing is comparing the probability distributions of each of the authors' cocitations.

The interpretation of cocitation profiles as probability distributions is especially interesting because it provides new insights into the question of what might be useful similarity measures for author cocitation analysis. It can now be seen that a natural approach to this question is to have a look at some well-known similarity measures for probability distributions. We first note that the use of the Pearson correlation or the cosine to measure the similarity between probability distributions is, to the best of our knowledge, very uncommon. For the Pearson correlation this is not surprising, since the Pearson correlation does not satisfy the basic requirements that any similarity measure for probability distributions should satisfy. (These are the requirements that the value of the similarity measure is maximal if and only if two distributions are identical and that it is minimal if and only if two distributions are non-overlapping.) The cosine does satisfy these requirements, but despite of this its use as a similarity measure for probability distributions seems rather unusual. Perhaps the most popular similarity measure for probability distributions is the Kullback-Leibler distance from the field of information theory. However, this similarity measure has difficulties with zero probabilities and hence with zero cocitation counts. As a consequence, the measure is not very useful for author cocitation analysis. The Jensen-Shannon distance [4], which is closely related to the Kullback-Leibler distance, does 
not have these difficulties and is therefore more interesting from the point of view of author cocitation analysis. ${ }^{2}$ Based on the Jensen-Shannon distance, the similarity between authors $i$ and $j$ can be calculated as

$$
\mathrm{JS}(i, j)=1-\frac{1}{2}\left(\sum_{k \neq i, j} p_{i k} \log \frac{p_{i k}}{\bar{p}_{k}}\right)-\frac{1}{2}\left(\sum_{k \neq i, j} p_{j k} \log \frac{p_{j k}}{\bar{p}_{k}}\right),
$$

where the $\log$ arithm has base 2 and where $0 \log 0$ and $0 \log (0 / 0)$ are defined as 0 . Furthermore, $\bar{p}_{k}=\left(p_{i k}+p_{j k}\right) / 2$. Another well-known similarity measure for probability distributions is the Bhattacharyya distance [2]. This is a popular similarity measure in pattern recognition and related fields. Using the Bhattacharyya distance, the similarity between authors $i$ and $j$ is calculated as

$$
\mathrm{B}(i, j)=\sum_{k \neq i, j} \sqrt{p_{i k} p_{j k}} .
$$

$\mathrm{JS}(i, j)$ and $\mathrm{B}(i, j)$ both have a value between 0 and 1 . They have a value of 1 if and only if the probability distributions given by the $p_{i k} \mathrm{~s}$ and $p_{j k} \mathrm{~s}$ are identical, and they have a value of 0 if and only if these distributions are non-overlapping. In addition to the similarity measures mentioned above, there are a number of other similarity measures that are sometimes used to compare probability distributions. In the rest of this report, however, we focus our attention on the above-mentioned similarity measures.

\section{Practical relevance}

White [8] argues that theoretical shortcomings of the Pearson correlation are problematic only if there is a substantive difference between results based on the Pearson correlation and results based on theoretically sound similarity measures. We agree with this reasoning. However, contrary to White, we believe that such substantive differences do indeed exist. To show the existence of these differences, we take a well-known author cocitation study by White and McCain [10] as an example. Among other things, White and McCain provide a multidimensional scaling map of the similarities between the top 100 authors in the field of information science in the period 1988-1995. They use the Pearson correlation to calculate the similarities between

\footnotetext{
${ }^{2}$ Both the Kullback-Leibler distance and the Jensen-Shannon distance are in fact measures of the dissimilarity between probability distributions. For the present discussion, the difference between similarity and dissimilarity measures is not important, and we therefore refer to all measures as similarity measures.
} 


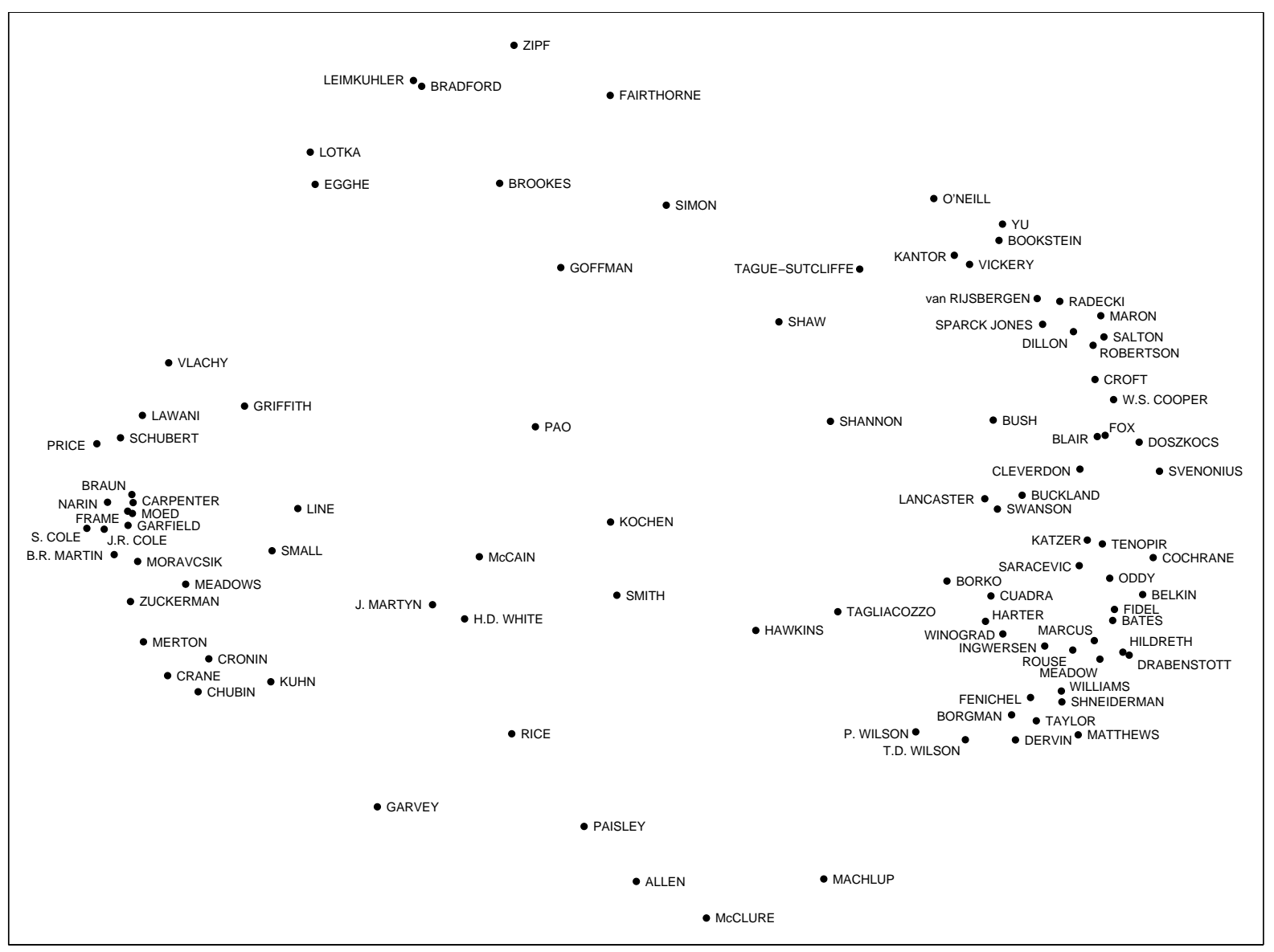

Figure 1: Map obtained using the Pearson correlation.

the authors. Using the ALSCAL program in SPSS, we replicated the analysis of White and McCain and obtained the map shown in Figure 1. This map is almost identical to the one provided by White and McCain. (Where the two maps are different, this is most likely due to slight differences in the way in which the cocitation data was collected and preprocessed.) In addition to the map in Figure 1, we constructed three more maps. In these maps, the similarities between the authors were calculated based on the cosine, the Jensen-Shannon distance, and the Bhattacharyya distance. The maps obtained using the cosine and the Jensen-Shannon distance are shown in Figures 2 and 3, respectively. The map obtained using the Bhattacharyya distance turned out to be almost identical to the map obtained using the Jensen-Shannon distance and is therefore not shown.

Comparing the map in Figure 1 with the maps in Figures 2 and 3, it is immediately apparent that there is a substantive difference between results based on the Pearson correlation and results based on theoretically sound similarity measures such as the cosine and the Jensen-Shannon 


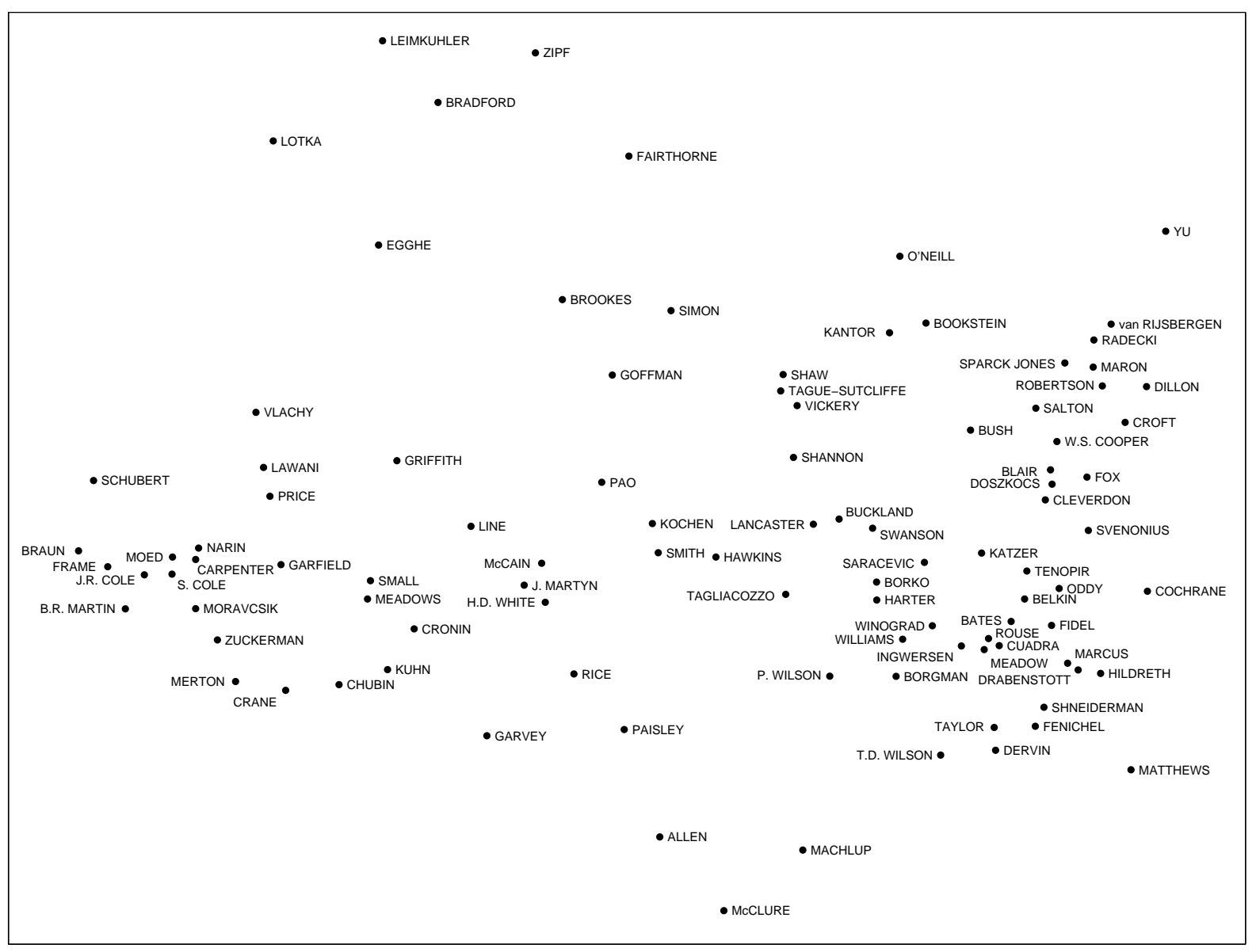

Figure 2: Map obtained using the cosine. 


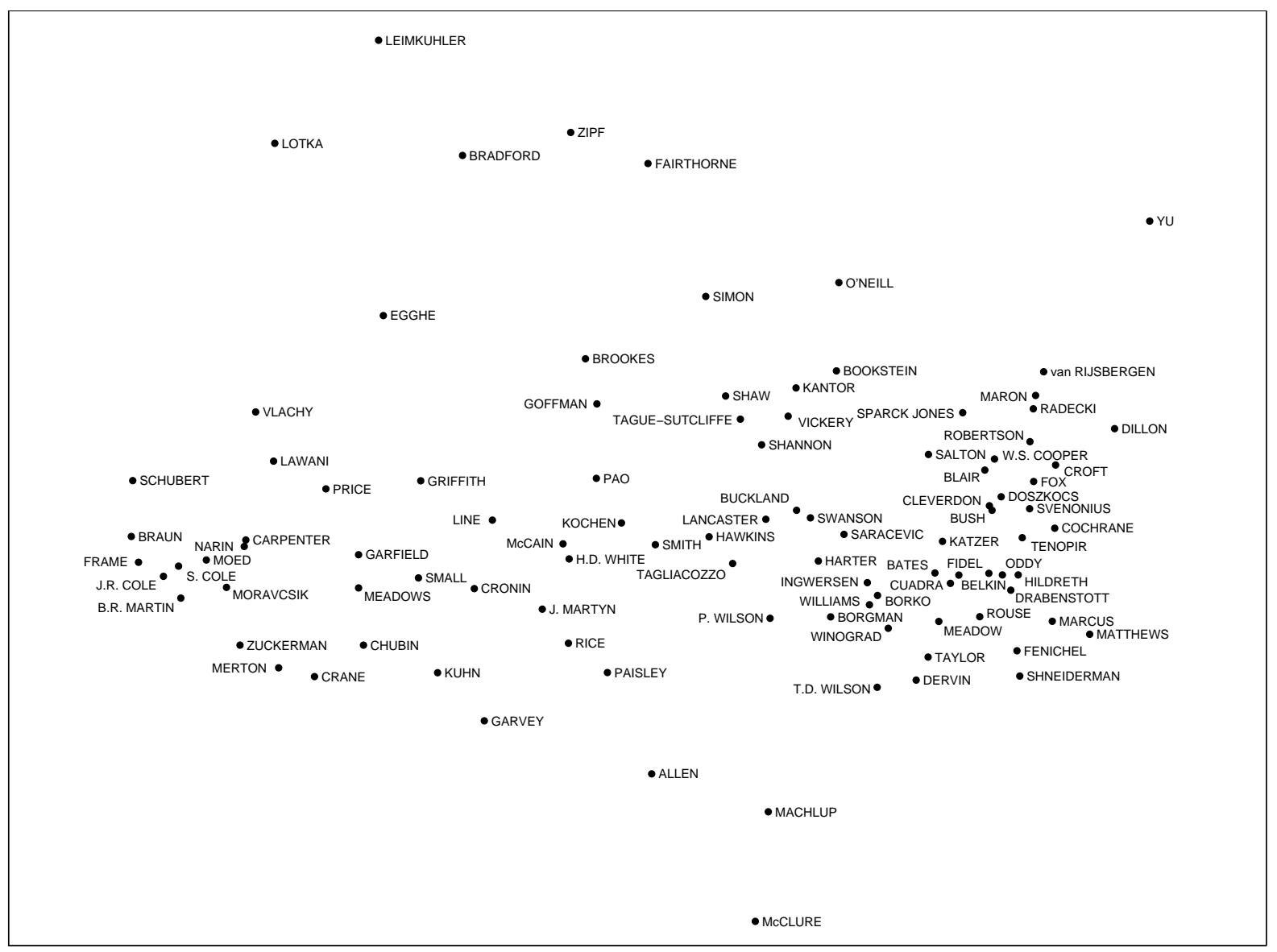

Figure 3: Map obtained using the Jensen-Shannon distance. 
distance. In the map in Figure 1, there is a clear division of the authors into two clusters, a cluster of domain analysis authors and a cluster of information retrieval authors. The clusters are located on opposite sides of the map, and only a small number of authors are located in between the clusters. Hence, based on the map in Figure 1, information science appears to be a field consisting of two subfields, domain analysis and information retrieval, that are almost completely separated from each other. Now consider the maps in Figures 2 and 3. In these maps, the clustering of authors is either much less pronounced than in the map in Figure 1 or there is no clustering at all. Although a number of typical domain analysis authors are located in the far left part of the maps in Figures 2 and 3 and a number of typical information retrieval authors in the far right part, many authors are located somewhere in between the extremes. Consequently, based on these maps, information science appears to be a fairly unified field with a substantial number of connections between its two main subfields, domain analysis and information retrieval. This is a very different picture of the information science field than the picture that emerges from the map in Figure 1.

There are two final remarks that we would like to make. Both remarks are based on a paper by White [9] in which he uses pathfinder networks to perform an author cocitation analysis of the information science field. First, White ([9], p. 427) does not seem to be completely satisfied with the maps of the information science field provided in [10]. In particular, he expresses some concerns about the "empty centers" that appear in these maps (also visible in the map in Figure 1). He further notes that the appearance of "empty centers" is not confined to information science but also happens when mapping other heterogeneous fields. White seems to prefer maps based on pathfinder networks because such maps do not have "empty centers". Interestingly, our results seem to indicate that the issue of the "empty centers" can simply be resolved by using a theoretically sound similarity measure, such as the cosine or the Jensen-Shannon distance, instead of the Pearson correlation. Our second and related remark is concerned with White's statement that 'the 'empty center' should be recognized as a metaphor growing out of the (multidimensional scaling) mapping algorithm" ([9], p. 427). Our results point in a different direction. The issue of the "empty centers" seems to be caused by the use of the Pearson correlation as a similarity measure for cocitation profiles rather than by the use of multidimensional scaling as a mapping technique for author similarities. 


\section{Conclusion}

We have argued that the Pearson correlation has some shortcomings as a measure of the similarity between cocitation profiles. As a consequence, the use of the Pearson correlation in author cocitation analysis is, in our opinion, not very satisfactory. The cosine does not have the shortcomings of the Pearson correlation, and we therefore regard it as a more appropriate similarity measure for cocitation profiles. The interpretation of cocitation profiles as probability distributions suggests other similarity measures that may be useful for author cocitation analysis. In particular, similarity measures based on the Jensen-Shannon distance or the Bhattacharyya distance may be considered. In an author cocitation study of the field of information science, the Pearson correlation gives results that are quite different from results obtained using theoretically sound similarity measures. This shows that our findings are not merely of theoretical interest but also have a high practical relevance.

\section{References}

[1] P. Ahlgren, B. Jarneving, and R. Rousseau. Requirements for a cocitation similarity measure, with special reference to Pearson's correlation coefficient. Journal of the American Society for Information Science and Technology, 54(6):550-560, 2003.

[2] A. Bhattacharyya. On a measure of divergence between two statistical populations defined by their probability distributions. Bulletin of the Calcutta Mathematical Society, 35:99109, 1943.

[3] L. Leydesdorff and L. Vaughan. Co-occurrence matrices and their applications in information science: Extending ACA to the web environment. Journal of the American Society for Information Science and Technology, 57(12):1616-1628, 2006.

[4] J. Lin. Divergence measures based on the Shannon entropy. IEEE Transactions on Information Theory, 37(1):145-151, 1991.

[5] K. McCain. Mapping authors in intellectual space: A technical overview. Journal of the American Society for Information Science, 41(6):433-443, 1990. 
[6] J. Schneider and P. Borlund. Matrix comparison, part 1: Motivation and important issues for measuring the resemblance between proximity measures or ordination results. Journal of the American Society for Information Science and Technology, 58(11):1586-1595, 2007.

[7] L. Waltman and N. Van Eck. Some comments on the question whether co-occurrence data should be normalized. Journal of the American Society for Information Science and Technology, 58(11):1701-1703, 2007.

[8] H. White. Author cocitation analysis and Pearson's $r$. Journal of the American Society for Information Science and Technology, 54(13):1250-1259, 2003.

[9] H. White. Pathfinder networks and author cocitation analysis: A remapping of paradigmatic information scientists. Journal of the American Society for Information Science and Technology, 54(5):423-434, 2003.

[10] H. White and K. McCain. Visualizing a discipline: An author co-citation analysis of information science, 1972-1995. Journal of the American Society for Information Science, 49(4):327-355, 1998. 


\section{Publications in the Report Series Research ${ }^{*}$ in Management}

\section{ERIM Research Program: "Business Processes, Logistics and Information Systems"}

2007

India: a Case of Fragile Wireless Service and Technology Adoption?

L-F Pau and J. Motiwalla

ERS-2007-011-LIS

http://hdl.handle.net/1765/9043

Some Comments on the Question Whether Co-occurrence Data Should Be Normalized

Ludo Waltman and Nees Jan van Eck

ERS-2007-017-LIS

http://hdl.handle.net/1765/9401

Extended Producer Responsibility in the Aviation Sector

Marisa P. de Brito, Erwin A. van der Laan and Brijan D. Irion

ERS-2007-025-LIS

http://hdl.handle.net/1765/10068

Logistics Information and Knowledge Management Issues in Humanitarian Aid Organizations

Erwin A. van der Laan, Marisa P. de Brito and S. Vermaesen

ERS-2007-026-LIS

http://hdl.handle.net/1765/10071

Bibliometric Mapping of the Computational Intelligence Field

Nees Jan van Eck and Ludo Waltman

ERS-2007-027-LIS

http://hdl.handle.net/1765/10073

Approximating the Randomized Hitting Time Distribution of a Non-stationary Gamma Process

J.B.G. Frenk and R.P. Nicolai

ERS-2007-031-LIS

http://hdl.handle.net/1765/10149

Application of a General Risk Management Model to Portfolio Optimization Problems with Elliptical Distributed Returns for Risk Neutral and Risk Averse Decision Makers

Bahar Kaynar, S. Ilker Birbil and J.B.G. Frenk

ERS-2007-032-LIS

http://hdl.handle.net/1765/10151

Optimal Zone Boundaries for Two-class-based Compact 3D AS/RS

Yugang Yu and M.B.M. de Koster

ERS-2007-034-LIS

http://hdl.handle.net/1765/10180

Portfolios of Exchange Relationships: An Empirical Investigation of an Online Marketplace for IT Services

Uladzimir Radkevitch, Eric van Heck and Otto Koppius

ERS-2007-035-LIS

http://hdl.handle.net/1765/10072

From Closed-Loop to Sustainable Supply Chains: The WEEE case

J. Quariguasi Frota Neto, G. Walther, J.Bloemhof, J.A.E.E van Nunen and T.Spengler

ERS-2007-036-LIS

http://hdl.handle.net/1765/10176 
A Methodology for Assessing Eco-Efficiency in Logistics Networks

J. Quariguasi Frota Neto, G. Walther, J.Bloemhof, J.A.E.E van Nunen and T.Spengler

ERS-2007-037-LIS

http://hdl.handle.net/1765/10177

Strategic and Operational Management of Supplier Involvement in New Product Development: a Contingency Perspective Ferrie E.A. van Echtelt, Finn Wynstra and Arjan J. van Weele

ERS-2007-040-LIS

http://hdl.handle.net/1765/10456

How Will Online Affiliate Marketing Networks Impact Search Engine Rankings?

David Janssen and Eric van Heck

ERS-2007-042-LIS

http://hdl.handle.net/1765/10458

Modelling and Optimizing Imperfect Maintenance of Coatings on Steel Structures

R.P. Nicolai, J.B.G. Frenk and R. Dekker

ERS-2007-043-LIS

http://hdl.handle.net/1765/10455

Human Knowledge Resources and Interorganizational Systems

Mohammed Ibrahim, Pieter Ribbers and Bert Bettonvil

ERS-2007-046-LIS

http://hdl.handle.net/1765/10457

Revenue Management and Demand Fulfilment: Matching Applications, Models, and Software

Rainer Quante, Herbert Meyr and Moritz Fleischmann

ERS-2007-050-LIS

http://hdl.handle.net/1765/10464

Mass Customization in Wireless Communication Services: Individual Service Bundles and Tariffs

Hong Chen and Louis-Francois Pau

ERS-2007-051-LIS

http://hdl.handle.net/1765/10515

Individual Tariffs for Mobile Services: Analysis of Operator Business and Risk Consequences

Hong Chen and Louis-Francois Pau

ERS-2007-052-LIS

http://hdl.handle.net/1765/10516

Individual Tariffs for Mobile Services: Theoretical Framework and a Computational Case in Mobile Music

Hong Chen and Louis-Francois Pau

ERS-2007-053-LIS

http://hdl.handle.net/1765/10517

Individual Tariffs for Mobile Communication Services

Hong Chen and Louis-Francois Pau

ERS-2007-054-LIS

http://hdl.handle.net/1765/10518

Is Management Interdisciplinary? The Evolution of Management as an Interdisciplinary Field of Research and Education in the Netherlands

Peter van Baalen and Luchien Karsten

ERS-2007-047-LIS

http://hdl.handle.net/1765/10537 
Detecting and Forecasting Economic Regimes in Multi-Agent Automated Exchanges

Wolfgang Ketter, John Collins, Maria Gini, Alok Gupta and Paul Schrater

ERS-2007-065-LIS

http://hdl.handle.net/1765/10594

Emergency Messaging to General Public via Public Wireless Networks

P.Simonsen and L-F Pau

ERS-2007-078-LIS

http://hdl.handle.net/1765/10718

Flexible Decision Control in an Autonomous Trading Agent

John Collins, Wolfgang Ketter and Maria Gini

ERS-2007-079-LIS

http://hdl.handle.net/1765/10719

Discovering the Dynamics of Smart Business Networks

L-F Pau

ERS-2007-081-LIS

http://hdl.handle.net/1765/10732

Performance Approximation and Design of Pick-and-Pass Order Picking Systems

Mengfei Yu and René de Koster

ERS-2007-082-LIS

http://hdl.handle.net/1765/10733

Distributed Services with Foreseen and Unforeseen Tasks: The Mobile Re-allocation Problem Jose Antonio Larco, Rommert Dekker and Uzay Kaymak ERS-2007-087-LIS

http://hdl.handle.net/1765/10773

Appropriate Similarity Measures for Author Cocitation Analysis

Nees Jan van Eck and Ludo Waltman

ERS-2007-091-LIS

http://hdl.handle.net/1765/10889

* A complete overview of the ERIM Report Series Research in Management: https://ep.eur.nl/handle/1765/1

ERIM Research Programs:

LIS Business Processes, Logistics and Information Systems

ORG Organizing for Performance

MKT Marketing

F\&A Finance and Accounting

STR Strategy and Entrepreneurship 